\title{
Therapeutic plasma exchange in patients with COVID-19 pneumonia in intensive care unit: a retrospective study
}

\author{
Bulent Gucyetmez ${ }^{1,2^{*}} \mathbb{D}$, Hakan Korkut Atalan ${ }^{3}$, Ibrahim Sertdemir ${ }^{4}$, Ulkem Cakir ${ }^{5}$, Lutfi Telci ${ }^{2,6,7}$ \\ and COVID-19 Study Group
}

In patients with COVID-19 pneumonia, high risk of thrombosis became a current issue, and D-dimer levels indicating fibrin degradation products (FDPs) in the plasma were found as a predictor for mortality $[1,2]$. Although unfractionated heparin (UFH) and lowmolecular-weight heparin (LMWH) decrease the production of FDPs by inhibiting factors Xa and II, they cannot contribute metabolization of existing FDPs. Furthermore, FDPs cannot be filtered by known cytokine filters because of their molecular weight (minimum 240 $\mathrm{kDa})[3,4]$. Yet, FDPs can be removed by therapeutic plasma exchange (TPE) [5]. Therefore, recently, three consecutive TPE sessions were performed in selected patients with COVID-19 pneumonia in intensive care units (ICUs) after the assessment of their clinical and coagulation status. In the study, the effect of TPE on outcomes was retrospectively investigated in patients with COVID19 pneumonia.

All COVID-19 patients admitted to 5 different tertiary ICUs between 10 March and 10 May 2020 were evaluated, and 73 of 91 patients were included in the study. The patients who died within the first 4 days and who were still in the ICUs on 10 May were excluded. According to the Turkish Health Minister Algorithm for COVID-19, all included patients received the same antiviral (favipiravir, hydroxychloroquine, azithromycin) therapy and anticoagulant prophylaxis (UFH infusion

\footnotetext{
* Correspondence: bulentgucyetmez@gmail.com

'Department of Anesthesiology and Reanimation, Acibadem Mehmet Ali

Aydinlar University School of Medicine, Kerem Aydınlar Kampüsü, Kayışdağı Cad No:32 Ataşehir, 34752 Istanbul, Turkey

${ }^{2}$ General Intensive Care Unit, Acibadem International Hospital, Istanbul, Turkey

Full list of author information is available at the end of the article
}

$100 \mathrm{mcg} / \mathrm{kg}$ or LMWH $0.01 \mathrm{~mL} / \mathrm{kg}$ ). Since two different protocols were used in 5 ICUs, patients with D-dimer $\geq 2$ in 3 ICUs had only received therapeutic anticoagulation whereas patients with D-dimer $\geq 2$ in the other 2 ICUs had received TPE plus therapeutic anticoagulation. In all ICUs, for all patients in GII, echocardiography, lower extremity venous Doppler, and, if pulmonary thrombosis suspected, thorax computerized tomography angiography were performed. After collecting data, 73 patients were divided into 2 groups as group I (GI) (D-dimer $<2$ $\mathrm{mg} / \mathrm{L}$ ) and group II (GII) (D-dimer $\geq 2 \mathrm{mg} / \mathrm{L}$ ), and then GII was also divided into 2 groups as GIIa (TPE+) and GIIb (TPE-). Patients' characteristics, respiratory and laboratory parameters, and outcomes were recorded. Propensity score matching (PSM) analysis was conducted on $\mathrm{R}$ v4.0.1 ( 0.2 caliper without replacement and nearest neighbor model, 1:1 ratio) by using 14 covariates (age, gender, CCI, APACHE II, SOFA score, lactate, leucocyte, lymphocyte, D-dimer and creatinine at the ICU admission, maximum respiratory support, the usage of steroid, IL-6 blocker, and cytokine filter).

The total mortality rate was $27.4 \%$. Mortality rates of GI and GII were 5\% and 35.9\%, respectively. In GII, major thromboembolic events were not detected in any patients. The median (min-max) day for the starting TPE was 3 (2-4). In GIIa, APACHE II, SOFA scores, Ddimer and interleukin-6 (IL-6) levels at the ICU admission, and length of ICU stay were significantly higher than those of GI whereas mortality rates were similar in those groups (Table 1). The median values of the LOSICU in survivors and non-survivors in GII were 14 (6.5$21.5)$ and $15.5(8-23)$, respectively $(p=0.630)$. In GIIa, lactate dehydrogenase (LDH), D-dimer, ferritin, IL-6, Creactive protein $(\mathrm{CRP})$, and procalcitonin levels were 
Table 1 Comparisons of patients groups

\begin{tabular}{|c|c|c|c|c|c|c|c|c|}
\hline & \multirow{3}{*}{$\begin{array}{l}\text { Gl }(\mathrm{D}-\text { dimer }<2) \\
(n=20)\end{array}$} & \multicolumn{7}{|c|}{ Gll (D-dimer $\geq 2$ ) } \\
\hline & & \multicolumn{4}{|l|}{ Before PSM } & \multicolumn{3}{|l|}{ After PSM } \\
\hline & & $\begin{array}{l}\text { Glla (TPE+) } \\
(n=18)\end{array}$ & $\begin{array}{l}\text { Gllb (TPE-) } \\
(n=35)\end{array}$ & $\begin{array}{l}\mathrm{p}_{1}(\mathrm{Gl} \\
\text { and Glla) }\end{array}$ & $\begin{array}{l}\mathrm{p}_{2} \text { (Glla } \\
\text { and Gllb) }\end{array}$ & $\begin{array}{l}\text { Glla (TPE+) } \\
(n=12)\end{array}$ & $\begin{array}{l}\text { Gllb (TPE-) } \\
(n=12)\end{array}$ & $\begin{array}{l}\mathrm{p}_{2}(\mathrm{G} l l a \\
\text { and Gllb) }\end{array}$ \\
\hline Age (years) & $60 \pm 14$ & $62 \pm 12$ & $62 \pm 15$ & 0.615 & 0.951 & $61 \pm 14$ & $64 \pm 17$ & 0.605 \\
\hline Male, $n(\%)$ & $13(65.0)$ & $14(77.8)$ & $26(74.3)$ & 0.386 & 0.780 & $8(66.7)$ & $8(66.7)$ & 1.000 \\
\hline $\mathrm{BMI}\left(\mathrm{kg} / \mathrm{m}^{2}\right)$ & $27.3(5.8)$ & $27.9(5.5)$ & $27.3(6.6)$ & 0.290 & 0.237 & $28.5(6.1)$ & $25.0(6.6)$ & 0.078 \\
\hline $\mathrm{CCl}$ & $2.5(4)$ & $3(3)$ & $4(3)$ & 0.919 & 0.422 & $3.0 \pm 2.2$ & $3.8 \pm 1.7$ & 0.270 \\
\hline \multicolumn{9}{|l|}{ At the ICU admission } \\
\hline APACHE II & $12 \pm 4$ & $17 \pm 4$ & $17 \pm 5$ & 0.002 & 0.886 & $17 \pm 3.3$ & $17.5 \pm 5.6$ & 0.794 \\
\hline SOFA Score & $5(3)$ & $6(1)$ & $7(3)$ & 0.002 & 0.223 & $6(2)$ & $6(2)$ & 0.713 \\
\hline $\mathrm{PaO}_{2} / \mathrm{FiO}_{2}$ ratio & $128(68)$ & $97(51)$ & $113(79)$ & 0.251 & 0.229 & $108(106)$ & $125(103)$ & 0.551 \\
\hline $\mathrm{SpO}_{2}(\%)$ & $89(5)$ & $91(7)$ & $89(5)$ & 0.377 & 0.597 & $92(10)$ & $91(5)$ & 0.590 \\
\hline Lactate $(\mathrm{mmol} / \mathrm{L})$ & $1.4(0.6)$ & $1.4(0.7)$ & $1.4(0.9)$ & 0.988 & 0.631 & $1.5(0.8)$ & $1.3(0.5)$ & 0.291 \\
\hline WBC $\left(\times 10^{3} / \mu \mathrm{L}\right)$ & $9.6(3.9)$ & $6.9(6.4)$ & $8.2(6.5)$ & 0.573 & 0.353 & $8.7 \pm 4.9$ & $7.4 \pm 2.7$ & 0.430 \\
\hline Lymc $\left(\times 10^{3} / \mu \mathrm{L}\right)$ & $0.82 \pm 0.40$ & $0.80 \pm 0.34$ & $0.89 \pm 0.42$ & 0.553 & 0.271 & $0.83 \pm 0.3$ & $0.82 \pm 0.5$ & 0.963 \\
\hline D-dimer $(\mathrm{mg} / \mathrm{L})^{\&}$ & $1.2(0.3-1.9)$ & $5.0(2.1-35.2)$ & $7.2(2.1-35.5)$ & $<0.001$ & 0.151 & $4.5(2.1-35.2)$ & $6.0(2.2-32.2)$ & 0.514 \\
\hline Ferritin (ng/mL) & 1015 (1735) & 1735 (1853) & $900(1454)$ & 0.158 & 0.018 & $1742(2117)$ & 605 (1346) & 0.012 \\
\hline IL-6 (pg/mL) $)^{\&}$ & $\begin{array}{l}28.3(5.3- \\
1418)^{(8)}\end{array}$ & $\begin{array}{l}134(36.2- \\
2958)^{(13)}\end{array}$ & $\begin{array}{l}254(33- \\
5233)^{(13)}\end{array}$ & 0.036 & 0.101 & $\begin{array}{l}155(39.6- \\
2958)^{(8)}\end{array}$ & $\begin{array}{l}237(33- \\
4885)^{(4)}\end{array}$ & 0.933 \\
\hline $\mathrm{CRP}(\mathrm{mg} / \mathrm{dL})$ & $18.6 \pm 10.9$ & $22.2 \pm 12.1$ & $27.8 \pm 10.4$ & 0.340 & 0.086 & $19.2 \pm 10.3$ & $24.0 \pm 11.0$ & 0.275 \\
\hline Creatinine $(\mathrm{mg} / \mathrm{dL})$ & $0.88(0.29)$ & $0.87(0.37)$ & $0.99(0.82)$ & 0.874 & 0.051 & $0.91 \pm 0.3$ & $0.90 \pm 0.3$ & 0.944 \\
\hline Urea (mg/dL) & $28(29)$ & $32(19)$ & $36(26)$ & 0.942 & 0.288 & $28(32)$ & $35(14)$ & 0.291 \\
\hline $\begin{array}{l}\text { Number of damaged } \\
\text { lobes, } n(\%)^{\&}\end{array}$ & $3(2-4)$ & $3(2-5)$ & $3(2-5)$ & 0.149 & 0.118 & $3(2-5)$ & $3(3-5)$ & 0.671 \\
\hline \multicolumn{9}{|l|}{ In the first $48 \mathrm{~h}$} \\
\hline Breath rate/min (max) & $34(6)$ & $33(9)$ & $33(5)$ & 0.988 & 0.713 & $33(11)$ & $33(5)$ & 0.590 \\
\hline $\mathrm{PaO}_{2} / \mathrm{FiO}_{2}$ ratio (min) & $117 \pm 42$ & $98 \pm 30$ & $105 \pm 34$ & 0.087 & 0.376 & $104 \pm 32.4$ & $120 \pm 32.5$ & 0.235 \\
\hline $\mathrm{FiO}_{2}(\%)(\max )$ & $75(48)$ & $80(30)$ & $80(35)$ & 0.082 & 0.969 & $80(25)$ & $80(30)$ & 0.799 \\
\hline PEEP $\left(\mathrm{cmH}_{2} \mathrm{O}\right)(\max )$ & $12(6)$ & $12(4)$ & $14(4)$ & 0.502 & 0.056 & $12.0 \pm 2.3$ & $13.0 \pm 1.9$ & 0.215 \\
\hline $\begin{array}{l}\mathrm{C}_{\mathrm{dyn}}\left(\mathrm{ml} / \mathrm{cmH}_{2} \mathrm{O}\right) \\
(\mathrm{min})\end{array}$ & $44(6)$ & $37(12)$ & $41(8)$ & 0.003 & 0.058 & $36.3 \pm 6.6$ & $39.5 \pm 7.0$ & 0.265 \\
\hline \multicolumn{9}{|l|}{ In the first week } \\
\hline WBC $\left(\times 10^{3} / \mu \mathrm{L}\right)(\max )$ & $13.2(5.8)$ & $11.0(8.9)$ & $12.6(6.6)$ & 0.077 & 0.086 & $10.4(10.3)$ & $11.0(6.7)$ & 0.590 \\
\hline $\operatorname{WBC}\left(\times 10^{3} / \mu \mathrm{L}\right)(\mathrm{min})$ & $5.9(2)$ & $6.3(4)$ & $4.9(4)$ & 0.718 & 0.612 & $6.7(4.4)$ & $4.6(1.5)$ & 0.219 \\
\hline Lymc $\left(\times 10^{3} / \mu \mathrm{L}\right)(\mathrm{min})$ & $0.48(0.40)$ & $0.5(0.28)$ & $0.49(0.46)$ & 0.919 & 0.573 & $0.52(0.29)$ & $0.45(0.28)$ & 0.551 \\
\hline NLCR (max) & $16.4(16.2)$ & $15(8)$ & $11(11)$ & 0.460 & 0.517 & $13.6(10.1)$ & $11.6(11.5)$ & 0.843 \\
\hline $\begin{array}{l}\text { Lactate }(\mathrm{mmol} / \mathrm{L}) \\
\text { (max) }\end{array}$ & $2.1(0.7)$ & $2.4(1.1)$ & $2.4(0.8)$ & 0.087 & 0.955 & $2.3(1.0)$ & $2.4(1.6)$ & 0.347 \\
\hline Fluid balance $(\mathrm{mL})$ & $3670(3198)$ & $4552(2973)$ & 3849 (2196) & 0.874 & 0.441 & $4174 \pm 2907$ & $5331 \pm 3170$ & 0.361 \\
\hline $\begin{array}{l}\text { Total fluid (mL/kg/ } \\
\text { day) }\end{array}$ & $40.7(9.3)$ & $44.3(15.5)$ & $44.8(11)$ & 0.696 & 0.910 & $44.8 \pm 13.5$ & $48.7 \pm 12.0$ & 0.460 \\
\hline \multicolumn{9}{|c|}{ Respiratory support (max), $n$ (\%) } \\
\hline IMV & $13(65.0)$ & $16(88.8)$ & $30(85.7)$ & 0.084 & 0.746 & 11 (91.7) & $12(100)$ & 0.307 \\
\hline NIMV & $3(15.0)$ & $1(5.6)$ & $3(8.6)$ & 0.344 & 0.694 & $1(8.3)$ & 0 & 0.307 \\
\hline HFOT & $4(20.0)$ & $1(5.6)$ & $2(5.7)$ & 0.188 & 0.981 & 0 & 0 & NA \\
\hline
\end{tabular}


Table 1 Comparisons of patients groups (Continued)

\begin{tabular}{|c|c|c|c|c|c|c|c|c|}
\hline & \multirow{3}{*}{$\begin{array}{l}\text { Gl }(\mathrm{D}-\text { dimer < }) \\
(n=20)\end{array}$} & \multicolumn{7}{|c|}{ Gll (D-dimer $\geq 2$ ) } \\
\hline & & \multicolumn{4}{|l|}{ Before PSM } & \multicolumn{3}{|l|}{ After PSM } \\
\hline & & $\begin{array}{l}\text { Glla (TPE+) } \\
(n=18)\end{array}$ & $\begin{array}{l}\text { Gllb (TPE-) } \\
(n=35)\end{array}$ & $\begin{array}{l}\mathrm{p}_{1}(\mathrm{Gl} \\
\text { and Glla) }\end{array}$ & $\begin{array}{l}\mathrm{p}_{2} \text { (Glla } \\
\text { and Gllb) }\end{array}$ & $\begin{array}{l}\text { Glla (TPE }+) \\
(n=12)\end{array}$ & $\begin{array}{l}\text { Gllb (TPE-) } \\
(n=12)\end{array}$ & $\begin{array}{l}\mathrm{p}_{2} \text { (Glla } \\
\text { and Gllb) }\end{array}$ \\
\hline \multicolumn{9}{|l|}{$\begin{array}{l}\text { Additional therapies, } \\
n(\%)\end{array}$} \\
\hline Cytokine filters & $1(5.0)$ & $3(16.7)$ & $3(8.1)$ & 0.427 & 0.434 & $2(16.7)$ & $1(8.3)$ & 0.592 \\
\hline IL-6 blocker & $12(60.0)$ & $9(50.0)$ & $20(57.1)$ & 0.536 & 0.621 & $7(58.3)$ & $6(50)$ & 0.682 \\
\hline Steroids & $11(55.0)$ & $10(55.6)$ & $20(57.1)$ & 0.357 & 0.912 & $7(58.3)$ & $7(58.3)$ & 1.000 \\
\hline Duration of IMV $(h)^{\&}$ & $168(0-816)$ & $286(0-1008)$ & $192(0-720)$ & 0.112 & 0.067 & $316 \pm 271$ & $278 \pm 139$ & 0.671 \\
\hline $\mathrm{AKI}, n(\%)$ & $7(35.0)$ & $6(33.3)$ & $19(54.3)$ & 0.914 & 0.148 & $3(25)$ & $6(50)$ & 0.206 \\
\hline $\begin{array}{l}\text { Tracheotomized } \\
\text { patients, } n(\%)\end{array}$ & $2(10.0)$ & $2(11.1)$ & $1(2.9)$ & 0.911 & 0.218 & $1(8.3)$ & $0(0)$ & 0.307 \\
\hline LOS-ICU, (days) $)^{\&}$ & $12(6-34)$ & $20(5-42)$ & $11(7-35)$ & 0.017 & 0.003 & $20 \pm 10$ & $14 \pm 5$ & 0.067 \\
\hline Mortality, n (\%) & $1(5.0)$ & $3(16.7)$ & $16(45.7)$ & 0.242 & 0.037 & $1(8.3)$ & $7(58.3)$ & 0.009 \\
\hline
\end{tabular}

AKI acute kidney injury, APACHE /l Acute Physiology and Chronic Health Evaluation, BMI body mass index, $C C l$ Charlson comorbidity index, $C_{d y n}$ dynamic compliance, CRP C-reactive protein, HFOT high-flow oxygen therapy, ICU intensive care unit, IL-6 interleukin-6, IMV invasive mechanical ventilation, LOS length of stay, Lymc lymphocyte count, NIMV non-invasive mechanical ventilation, NLCR neutrophil-lymphocyte count ratio, PSM propensity score matching, SOFA, sequential organ failure assessment, TPE therapeutic plasma exchange, WBC white blood cell. Results were given as percentage, mean \pm sd, and median (IQR or min-max). ${ }^{\&}$ Minimum and maximum values. Student $t$ and Mann-Whitney $U$ tests were used for statistical analysis

significantly decreased after three consecutive TPEs (Table 2). Furthermore, although ferritin level at the ICU admission was higher in GIIa, the mortality rate in both before and after PSM was higher in GIIb $(45.7 \%$ and $58.3 \%)$ than in GIIa (16.7\% and 8.3\%) $(p=0.037, p=$ 0.009 , respectively) (Table 1 ).

Some patients with COVID-19 pneumonia have a high risk of thrombosis leading to worse outcomes. Therefore, monitoring D-dimer levels is crucial. In these groups of patients, TPE seems to be a treatment which may improve outcomes by effectively removing FDPs and restoring coagulation status. We are aware that TPE

Table 2 Comparisons of laboratory parameters in pre and postTPE procedure

\begin{tabular}{llll}
\hline & Pre-TPE & Post-TPE & $p$ \\
\hline WBC $\left(\times 10^{3} / \mu \mathrm{L}\right)$ & $9.08 \pm 4.1$ & $9.14 \pm 3.5$ & 0.951 \\
Neuc $\left(\times 10^{3} / \mu \mathrm{L}\right)$ & $7.38 \pm 3.1$ & $7.33 \pm 3.3$ & 0.953 \\
Lymc $\left(\times 10^{3} / \mu \mathrm{L}\right)$ & $0.9(0.5-1.3)$ & $1.02(0.77-1.27)$ & 0.053 \\
NLCR & $6.8(1.8-11.7)$ & $6.7(4.2-9.2)$ & 0.184 \\
LDH $(I \mathrm{U} / \mathrm{L})$ & $436(322-550)$ & $239(181-297)$ & $\mathbf{0 . 0 0 1}$ \\
D-dimer $(\mathrm{mg} / \mathrm{L})^{\&}$ & $7.8(2.1-35.2)$ & $1.3(0.6-3.9)$ & $<\mathbf{0 . 0 0 1}$ \\
Ferritin $(\mathrm{ng} / \mathrm{mL})^{\&}$ & $1268(399-6110)$ & $405(157-1650)$ & $\mathbf{0 . 0 0 1}$ \\
IL-6 $(\mathrm{pq} / \mathrm{mL})^{(13) \&}$ & $161(36.2-2958)$ & $24.5(1.5-130)$ & $\mathbf{0 . 0 0 1}$ \\
CRP $(\mathrm{mg} / \mathrm{dL})^{\&}$ & $11.8(0.4-29.7)$ & $0.9(0.3-7.2)$ & $<\mathbf{0 . 0 0 1}$ \\
Procalcitonin $(\mathrm{ng} / \mathrm{mL})^{\&}$ & $0.27(0.02-87)$ & $0.1(0.01-39)$ & $\mathbf{0 . 0 0 2}$ \\
\hline
\end{tabular}

CRP C-reactive protein, IL-6 interleukin-6, LDH lactate dehydrogenase, LymC lymphocyte count, Neuc neutrophil count, NLCR neutrophil-lymphocyte count ratio, TPE therapeutic plasma exchange, WBC white blood cell. Results were given as percentage, mean $\pm s d$, and median (quartiles or min-max).

${ }^{\&}$ Minimum and maximum values. Paired sample and Wilcoxon tests were used for the statistical analysis may not be routinely required in these patients [6]. However, we think that it should be featured as a part of the treatment especially in COVID-19 pneumonia patients with a high risk of thrombosis.

\section{Acknowledgements}

We would like to express our gratitude to our nurses who have taken care of the patients and performed TPE procedure in the ICU and COVID-19 Study Group. COVID-19 Group collaborating authors: Aylin Ogan², Aylin Cimet Ayyildiz$^{2}$, Berrin Yalcin², Behiye Oren ${ }^{6}$, Fadıl Havas ${ }^{6}$, Sevda Dizi ${ }^{6}$, Birsen Kose ${ }^{6}$, Umran Yakici, Cenk Sahan ${ }^{6}$, Elif Ozkilitci ${ }^{6}$, Ugur Tunali ${ }^{6}$, Deniz Gunes ${ }^{7}$, Ozlem Dincer ${ }^{7}$, Reyhan Sahin ${ }^{7}$, Duran Ozdemir ${ }^{7}$, Mehtap Selcuk ${ }^{8}$, Ceyhun Solakoglu ${ }^{8}$, Unsal Arif Turan ${ }^{8}$, Erkan Kaya ${ }^{8}$, Mustafa Emre Kavlak ${ }^{3}$, Pelin Katar ${ }^{3}$, Hande Aygun ${ }^{9}$, Kerim Cikim ${ }^{10}$, Ozkan Uysal ${ }^{10}$, Nur Ozturk Kaskir ${ }^{11}$, Aysun Soylu ${ }^{12}$ ${ }^{2}$ General Intensive Care Unit, Acibadem International Hospital, Istanbul, Turkey

${ }^{3}$ General Intensive Care Unit, Memorial Atasehir Hospital, Istanbul, Turkey ${ }^{6}$ General Intensive Care Unit, Acibadem Atakent Hospital, Istanbul, Turkey ${ }^{7}$ General Intensive Care Unit, Acibadem Bakirkoy Hospital, Istanbul, Turkey ${ }^{8}$ General Intensive Care Unit, Acibadem Kadikoy Hospital, Istanbul, Turkey ${ }^{9}$ Department of Infection Disease and Clinical Microbiology, Acibadem International Hospital, Istanbul, Turkey

${ }^{10}$ Department of Internal Medicine, Acibadem International Hospital, Istanbul, Turkey

${ }^{11}$ Department of Chest Disease, Acibadem International Hospital, Istanbul, Turkey

${ }^{12}$ Department of Biochemistry, Acibadem International Hospital, Istanbul, Turkey

Authors' contributions

BG: design of the work, analysis and interpretation of data, and writing. EO: acquisition of the data. HKA: acquisition and interpretation of the data and substantial contribution to the conception. UC and LT drafted the work. COVID-19 Study Group: acquisition of the data. The authors read and approved the final manuscript.

\section{Funding}

There was no funding for the research reported.

Availability of data and materials

All data was added as an Excel file in a supplementary information file. 


\section{Ethics approval and consent to participate}

The study was approved by The Scientific Committee of the Turkish Health Ministry (2020-05-11T22_01_29).

\section{Consent for publication}

No applicable

\section{Competing interests}

The authors declare that they have no competing interests.

\section{Author details}

'Department of Anesthesiology and Reanimation, Acibadem Mehmet Ali Aydinlar University School of Medicine, Kerem Aydınlar Kampüsü, Kayışdağı Cad No:32 Ataşehir, 34752 Istanbul, Turkey. ${ }^{2}$ General Intensive Care Unit, Acibadem International Hospital, Istanbul, Turkey. ${ }^{3}$ General Intensive Care Unit, Memorial Atasehir Hospital, Istanbul, Turkey. ${ }^{4}$ Department of Biostatistics and Bioinformatics, Institute of Health Sciences, Acibadem Mehmet Ali Aydinlar University, Istanbul, Turkey. ${ }^{5}$ Department of Nephrology, Acibadem Mehmet Ali Aydinlar University School of Medicine, Istanbul, Turkey. ${ }^{6}$ General Intensive Care Unit, Acibadem Atakent Hospital, Istanbul, Turkey. ${ }^{7}$ General Intensive Care Unit, Acibadem Bakirkoy Hospital, Istanbul, Turkey.

Received: 21 May 2020 Accepted: 28 July 2020

Published online: 08 August 2020

\section{References}

1. Helms J, Tacquard C, Severac F, Leonard-Lorant I, Ohana M, Delabranche X, et al. High risk of thrombosis in patients with severe SARS-CoV-2 infection: a multicenter prospective cohort study. Intensive Care Med. 2020;46:1089-98.

2. Zhang L, Yan X, Fan Q, Liu H, Liu X, Liu Z, et al. D-dimer levels on admission to predict in-hospital mortality in patients with Covid-19. J Thromb Haemost. 2020;18:1324-9.

3. Diab M, Platzer S, Guenther A, Sponholz C, Scherag A, Lehmann T, Velichkov I, Hagel S, Bauer M, Brunkhorst FM, et al. Assessing efficacy of CytoSorb haemoadsorber for prevention of organ dysfunction in cardiac surgery patients with infective endocarditis: REMOVE-protocol for randomised controlled trial. BMJ Open. 2020;10(3):e031912.

4. Walker JB, Nesheim ME. The molecular weights, mass distribution, chain composition, and structure of soluble fibrin degradation products released from a fibrin clot perfused with plasmin. J Biol Chem. 1999;274(8):5201-12.

5. Rimmer E, Houston BL, Kumar A, Abou-Setta AM, Friesen C, Marshall JC, Rock G, Turgeon AF, Cook DJ, Houston DS, et al. The efficacy and safety of plasma exchange in patients with sepsis and septic shock: a systematic review and meta-analysis. Crit Care. 2014;18(6):699.

6. Honore PM, Mugisha A, Kugener L, Redant S, Attou R, Gallerani A, De Bels D. Therapeutic plasma exchange as a routine therapy in septic shock and as an experimental treatment for COVID-19: we are not sure. Crit Care. 2020; 24(1):226.

\section{Publisher's Note}

Springer Nature remains neutral with regard to jurisdictional claims in published maps and institutional affiliations. 